\title{
Some Resolving Parameters in a Class of Cayley Graphs
}

\author{
Jia-Bao Liu $\mathbb{D D}^{1}$ and Ali Zafari $\mathbb{D}^{2}$ \\ ${ }^{1}$ School of Mathematics and Physics, Anhui Jianzhu University, Hefei 230601, China \\ ${ }^{2}$ Department of Mathematics, Faculty of Science, Payame Noor University, P.O. Box 19395-4697, Tehran, Iran
}

Correspondence should be addressed to Ali Zafari; zafari.math.pu@gmail.com

Received 7 July 2021; Revised 3 December 2021; Accepted 10 December 2021; Published 17 January 2022

Academic Editor: Ali Ahmad

Copyright (c) 2022 Jia-Bao Liu and Ali Zafari. This is an open access article distributed under the Creative Commons Attribution License, which permits unrestricted use, distribution, and reproduction in any medium, provided the original work is properly cited.

\begin{abstract}
Resolving parameters are a fundamental area of combinatorics with applications not only to many branches of combinatorics but also to other sciences. In this study, we construct a class of Toeplitz graphs and will be denoted by $T_{2 n}(W)$ so that they are Cayley graphs. First, we review some of the features of this class of graphs. In fact, this class of graphs is vertex transitive, and by calculating the spectrum of the adjacency matrix related with them, we show that this class of graphs cannot be edge transitive. Moreover, we show that this class of graphs cannot be distance regular, and because of the difficulty of the computing resolving parameters of a class of graphs which are not distance regular, we regard this as justification for our focus on some resolving parameters. In particular, we determine the minimal resolving set, doubly resolving set, and strong metric dimension for this class of graphs.
\end{abstract}

\section{Introduction}

The graphs in this paper are simple, undirected, and connected. An automorphism of a graph $\Gamma$ is a permutation $\varphi$ of the vertex set of $\Gamma$ with the property that, for any vertices $x$ and $y$, we have $x$ is adjacent to $y$ in $\Gamma$ if and only if $\varphi(x)$ is adjacent to $\varphi(y)$ in $\Gamma$. The set of all automorphisms of a graph $\Gamma$, with the operation of composition of permutations, is a permutation group on $V(\Gamma)$ and a subgroup of the symmetric group on $V(\Gamma)$. This is the automorphism group of $\Gamma$, denoted by $A u t(\Gamma)$. Suppose $\Gamma_{1}$ and $\Gamma_{2}$ are two graphs. If there is a bijection, say $\varphi$, from $V\left(\Gamma_{1}\right)$ to $V\left(\Gamma_{2}\right)$ so that $x$ is adjacent to $y$ in $\Gamma_{1}$ if and only if $\varphi(x)$ is adjacent to $\varphi(y)$ in $\Gamma_{2}$, then we say that $\Gamma_{1}$ is isomorphic to $\Gamma_{2}$. If we consider a graph $\Gamma$ as a network, then the network stability is very important to us, and especially, if graph $\Gamma$ is vertex transitive, that is, $A u t(\Gamma)$ acts transitively on $V(\Gamma)$, then the cost of studying the network will be very low, and hence, the network will be more stable. Consider a finite group $G$, and suppose $Q$ is a subset of $G$ so that it is closed under taking inverses and does not contain the identity; then, the Cayley graph $\Gamma=\operatorname{Cay}(G, Q)$ has vertex set $G$ and edge set $E(\Gamma)=\left\{\{x, y\} \mid x^{-1} y \in Q\right\}$. Thus, the studying of Cayley graphs is very useful because every Cayley graph is vertex transitive [1]. The distance between any pair $u, v \in V(\Gamma)$ of vertices of $\Gamma$ is the length of geodesic between $u$ and $v$, denoted by $d_{\Gamma}(u, v)$ or simply $d(u, v)$. A vertex $x \in V(\Gamma)$ is said to resolve a pair $u, v \in V(\Gamma)$ if $d(u, x) \neq d(v, x)$. Resolving parameters are a fundamental area of combinatorics with applications not only to many branches of combinatorics but also to other sciences. For an arranged subset $R=$ $\left\{r_{1}, r_{2}, \ldots, r_{m}\right\}$ of vertices in a connected graph $\Gamma$, the metric representation of a vertex $v$ in $\Gamma$ is the $m$-vector $r(v \mid R)=$ $\left(d\left(v, r_{1}\right), d\left(v, r_{2}\right), \ldots, d\left(v, r_{m}\right)\right)$ relative to $R$. Also, the subset $R$ is considered as the resolving set for $\Gamma$ if any pair of vertices of $\Gamma$ is distinguished by some vertices of $R$. A resolving set with least number of vertices is referred as metric basis for $\Gamma$ and the cardinality of such resolving set is known as metric dimension denoted by $\beta(\Gamma)$. The metric dimension of a graph $\Gamma$ is the least number of vertices in a set with the property that the list of distances from any vertex to those in the set uniquely identifies that vertex. The concept of the metric dimension in algebraic graph theory dates back to the 1970s. It was defined independently by Harary and Melter [2] and by Slater [3]. In recent years, a considerable literature has developed [4]. This concept has different applications in 
the areas of network discovery and verification [5]. For more details, see [6-9]. An $\left(\begin{array}{lll}n & x & n\end{array}\right)$ matrix $T=\left(t_{i j}\right)$ is called a Toeplitz matrix if $t_{i j}=t_{i+1, j+1}$ for each $i, j=1, \ldots, n-1$, see [10]. In fact, a Toeplitz matrix is a square matrix so that entries in every diagonal parallel to the main diagonal are equal, and hence, a Toeplitz matrix is determined by its first row and column. A simple undirected graph $\Gamma$ with vertex set $\{1, \ldots, n\}$ and its adjacency matrix $T=\left(t_{i j}\right)$ is called a Toeplitz graph if $T$ is the Toeplitz matrix. In this paper, we consider a class of Toeplitz graphs will be denoted by $T_{2 n}(W)$ so that they are Cayley graphs as follows.

Let $n$ be a fixed even integer is greater than or equal 4; also, let $[2 n]=\{1,2, \ldots, 2 n\}$ and $\left[x_{2 n}\right]=\left\{x_{1}, x_{2}, \ldots, x_{2 n}\right\}$ be corresponding sets so that $x_{i}=i$. Hence, we say that $x_{i}<x_{j}$ if $i<j$. Now, let $W_{1}=\left\{x_{1}, x_{3}, \ldots, x_{2 n-1}\right\}$ and $W_{2}=\left\{x_{n}\right\}$ be subsets of the set $\left[x_{2 n}\right]$, and let $W=W_{1} \cup W_{2}=$ $\left\{x_{1}, x_{3}, \ldots, x_{n}, \ldots, x_{2 n-1}\right\}$ be a refinement of union of the two sets $W_{1}$ and $W_{2}$ so that $1=x_{1}<x_{3}<\ldots<x_{n}<\ldots<x_{2 n-1}$. We can see that a graph with $2 n$ vertices so that the vertices are labelled by the set $\{1,2, \ldots, 2 n\}$ and the edge set:

$$
\left\{i j|i, j \in[2 n],| j-i \mid=x_{t} \quad \text { for some } x_{t} \in \mathrm{W}\right\},
$$

which is Toeplitz graph $T_{2 n}(W)$. For more result of the Toeplitz graphs, see $[11,12]$. Figure 1 shows the Toeplitz graph $T_{8}(1,3,4,5,7)$.

In particular, we can verify that the Toeplitz graph $T_{2 n}(W)$ which is defined already is isomorphic to the Cayley graph $\Lambda=\operatorname{Cay}\left(\mathbb{D}_{2 n}, \Psi\right)$, where

$$
\mathbb{D}_{2 n}=\left\langle a, b \mid a^{n}=b^{2}=1, b a=a^{n-1} b\right\rangle
$$

is the dihedral group of order $2 n$, and $\Psi=\left\{a b, a^{2} b\right.$, $\left.\ldots, a^{n-1} b, b\right\} \cup\left\{a^{n / 2}\right\}$ is an inverse closed subset of $\mathbb{D}_{2 n}-\{1\}$. Thus, the Toeplitz graph $T_{2 n}(W)$ is a vertex transitive. Also, for convenience, we can use the symbols in the Cayley graph $\Lambda=\operatorname{Cay}\left(\mathbb{D}_{2 n}, \Psi\right)$, instead of the symbols in the Toeplitz graph $T_{2 n}(W)$. Some metrics for a class of distance regular graphs is computed in $[13,14]$. On the contrary, because of the difficulty of the computing resolving parameters of a class of graphs which are not distance regular, we regard this as justification for our focus on some resolving parameters in the Cayley graph $\Lambda=\operatorname{Cay}\left(\mathbb{D}_{2 n}, \Psi\right)$. The important results of this study are presented in Sections 3.1 and 3.2. In Section 3.1, first, we will be determining the automorphism group of the Cayley graph $\Lambda=\operatorname{Cay}\left(\mathbb{D}_{2 n}, \Psi\right)$; also, we will show that the Cayley graph $\Lambda=\operatorname{Cay}\left(\mathbb{D}_{2 n}, \Psi\right)$ cannot be distance regular. In particular, we will prove that the Cayley graph $\Lambda=\operatorname{Cay}\left(\mathbb{D}_{2 n}, \Psi\right)$ cannot be edge transitive. Moreover, in Section 3.2, we will be computing some resolving parameters for this class of Cayley graphs.

\section{Definitions and Preliminaries}

Definition 1 (see [15]). A graph $\Gamma$ is edge transitive if its automorphism group acts transitively on $E(\Gamma)$.

Definition 2 (see [15]). A graph $\Gamma$ is 1-transitive or symmetric if its automorphism group acts transitively on the set of paths of length 1 or 1-arcs.

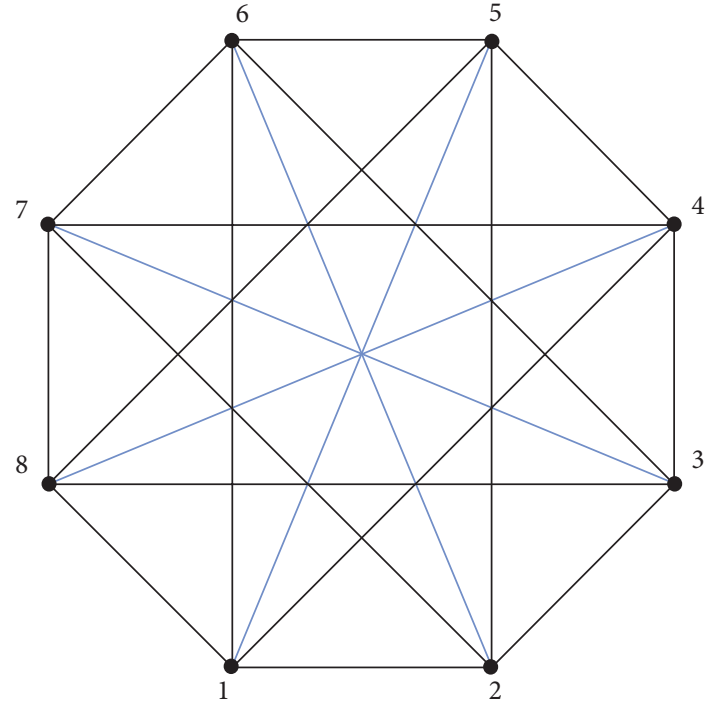

Figure 1: The Toeplitz graph $T_{8}(1,3,4,5,7)$.

Proposition 1 (see [15]). Let $\Gamma$ be a symmetric graph of valency $k$, and let $\lambda$ be a simple eigenvalue of $\Gamma$; then, $\lambda= \pm k$.

Definition 3 (see [16]). Suppose that $\Gamma$ is a regular graph of valency $k$ and for any two vertices $u$ and $v$ in $\Gamma$; if $d(u, v)=r$, then we have $\left|\Gamma_{r+1}(v) \cap \Gamma_{1}(u)\right|=b_{r}$, and $\left|\Gamma_{r-1}(v) \cap \Gamma_{1}(u)\right|=$ $c_{r}(0 \leq r \leq d)$. Then, we say that $\Gamma$ is a distance regular graph.

Proposition 2 (see [16]). If $\Gamma$ is a distance regular graph with diameter $d$, then $\Gamma$ has exactly $d+1$ distinct eigenvalues.

Definition 4 (see [17]). Suppose $\Gamma$ is a graph of order at least 2 ; vertices $x, y \in V(\Gamma)$ are said to doubly resolve vertices $u, v \in V(\Gamma)$ if $d(u, x)-d(u, y) \neq d(v, x)-d(v, y)$. A set $S=$ $\left\{s_{1}, s_{2}, \ldots, s_{l}\right\}$ of vertices of $\Gamma$ is a doubly resolving set of $\Gamma$ if every two distinct vertices of $\Gamma$ are doubly resolved by some two vertices of $S$. A doubly resolving set with minimum cardinality is called the minimal doubly resolving set. This minimum cardinality is denoted by $\psi(\Gamma)$.

Definition 5 (see [18]). Let $\Gamma$ be a graph. A vertex $w$ of $\Gamma$ strongly resolves two vertices $u$ and $v$ of $\Gamma$ if $u$ belongs to a shortest $v-w$ path or $v$ belongs to a shortest $u-w$ path. A set $S=\left\{s_{1}, s_{2}, \ldots, s_{m}\right\}$ of vertices of $\Gamma$ is a strong resolving set of $\Gamma$ if every two distinct vertices of $\Gamma$ are strongly resolved by some vertex of $S$. The strong metric dimension of a graph $\Gamma$ is the cardinality of smallest strong resolving set of $\Gamma$ and denoted by $s$ di $m(\Gamma)$.

\section{Main Results}

3.1. Some of the Features of the Cayley Graph Cay $\left(\mathbb{D}_{2 n}, \Psi\right)$. In this section, we review some of the features of the Cayley graph $\operatorname{Cay}\left(\mathbb{D}_{2 n}, \Psi\right)$. It is well known that the spectrum of a graph is the spectrum of the adjacency matrix related with it, that is, its set of eigenvalues together with their multiplicities. If all the eigenvalues of the adjacency matrix of a graph are 
integers, in this case, the graph related with it is called an integral graph, see [19]. As we shall see, the theory of integral graphs has connections to some parts of graph theory, edge transitivity, and symmetric graph. In the next theorem, we obtain the automorphism group of the Cayley graph $\operatorname{Cay}\left(\mathbb{D}_{2 n}, \Psi\right)$ by applications of wreath product in graph theory; for more details of the wreath product, see [20].

Proposition 3. Let $n$ be an even integer greater than or equal 4 , and $\Lambda=\operatorname{Cay}\left(\mathbb{D}_{2 n}, \Psi\right)$ be a Cayley graph on the dihedral group $\mathbb{D}_{2 n}$, where $\Psi$ is defined already. If $k=n / 2-1$, then Aut $(\Lambda) \cong \mathbb{Z}_{2} w r_{I} \operatorname{Sym}(k+1) w r_{I} \operatorname{Sym}(2), \quad$ where $I=\{1, \ldots, k+1\}$ and $J=\{1,2\}$.

Proof. We can see that the complement of $\Lambda$, denoted by $\bar{\Lambda}$, is isomorphic to the disjoint union of 2 copies of cocktail party graph $C P(n / 2)$, and we can show that $C P(n / 2)$ is isomorphic to the $\operatorname{Cay}\left(\mathbb{Z}_{n}, S_{k}\right)$, where $\mathbb{Z}_{n}$ is the cyclic group of order $n$ and $S_{k}=\{1, n-1,2, n-2, \ldots, k, n-k\}$, see Proposition 3.2 of [21]. Hence, given by the above discussion and the theorem in [22], we have Aut $(\bar{\Lambda}) \cong A u t(C P(n / 2))$ $\left.w r_{I} \operatorname{Sym}(2)=\mathbb{Z}_{2} w r_{I} \operatorname{Sym}(k+1)\right) w r_{J} \operatorname{Sym}(2)$. In particular, we have $\operatorname{Aut}(\bar{\Lambda})=\operatorname{Aut}(\Lambda)$ because a simple undirected graph and its complement have the same automorphism group.

Proposition 4. Let $n$ be an even integer greater than or equal 4 , and $\Lambda=\operatorname{Cay}\left(\mathbb{D}_{2 n}, \Psi\right)$ be a Cayley graph on the dihedral group $\mathbb{D}_{2 n}$, where $\Psi$ is defined already; then, $\Lambda$ cannot be a distance regular graph.

Proof. It is not hard to see that the diameter of $\Lambda$ is 2 and $\Lambda$ is not a bipartite graph, because $a^{n / 2} \in \Psi$. Now, by a similar way, which is done in proof of Proposition 11 in [23], we can show that the adjacency matrix spectrum of $\Lambda$ is $n+1,1-n, 1^{(n-2)},-1^{(n)}$, where the superscripts give the multiplicities of eigenvalues with multiplicity greater than one. Hence, $\Lambda$ has exactly four distinct eigenvalues. Moreover, based on Proposition 2, we know that if $\Lambda$ is a distance regular graph with diameter $d$, then $\Lambda$ has exactly $d+1$ distinct eigenvalues. Thus, $\Lambda$ cannot be a distance regular graph.

Proposition 5. Let $n$ be an even integer greater than or equal 4 and $\Lambda=\operatorname{Cay}\left(\mathbb{D}_{2 n}, \Psi\right)$ be a Cayley graph on the dihedral group $\mathbb{D}_{2 n}$, where $\Psi$ is defined already; then, $\Lambda$ cannot be an edge transitive graph.

Proof. By contradiction, suppose $\Lambda$ is an edge transitive graph. It is well known that a connected graph that is edge transitive and vertex transitive need not be 1-transitive. In particular, in p.59, 7.53 of [24], Tutte proved that if a connected graph, regular of odd valency, is both vertex and edge transitive, then it is 1-transitive. Thus, if $\Lambda$ is a edge transitive graph, then it must be $\Lambda$ is a 1-transitive graph because it is vertex transitive of odd valency $n+1$. On the contrary, based on Proposition 1, if $\lambda$ is a simple eigenvalue of a 1-transitive graph $\Lambda$, then $\lambda= \pm(n+1)$, which is not the case, see Proposition 4. This contradiction shows that $\Lambda$ cannot be an edge transitive graph.

3.2. Metric Dimension, Minimal Doubly Resolving set, and Strong Resolving Set of the Cayley Graph Cay $\left(\mathbb{D}_{2 n}, \Psi\right)$

Theorem 1. If $n$ is an even integer greater than or equal 4 and $\Lambda=\operatorname{Cay}\left(\mathbb{D}_{2 n}, \Psi\right)$ is a Cayley graph on the dihedral group $\mathbb{D}_{2 n}$, where $\Psi$ is defined already, then the metric dimension of $\Lambda$ is $n$.

Proof. Let $V(\Lambda)=V_{1} \cup V_{2}$, where $V_{1}=\left\{a, a^{2}, \ldots, a^{n}\right\}$ and $V_{2}=\left\{a b, a^{2} b, \ldots, a^{n} b\right\}$. For every pair of distinct vertices $x, y \in V(\Lambda)$, the length of a shortest path from $x$ to $y$ is $d(x, y)=1$ or 2 because the diameter of $\Lambda$ is 2 . In particular, if $R$ is an arranged subset of $V_{1}$ or $V_{2}$ in graph $\Lambda$ such that $|R| \leq n$, then we can show that $R$ is not a resolving set of $\Lambda$. Let $R=R_{1} \cup R_{2}$ be an arranged subset of vertices in graph $\Lambda$ such that $R_{1}$ is a subset of $V_{1}, R_{2}$ is a subset of $V_{2}$, and $\left|R_{1} \cup R_{2}\right|=n$. In the following cases, we can be concluded that the metric dimension of $\Lambda$ is $n$.

Case 1: if $\left|R_{1}\right| \neq\left|R_{2}\right|$, then we can assume that $\left|R_{1}\right|<\left|R_{2}\right|$. Hence, there is a pair of distinct vertices $u_{1}, u_{2} \in V(\Lambda)-R$, such that $u_{1}, u_{2} \in V_{1}-R_{1}$, and a shortest path from $u_{1}$ to $u_{2}$ is $d\left(u_{1}, u_{2}\right)=1$. Therefore, the metric representation of the vertices $u_{1}, u_{2} \in V(\Lambda)-R$ is the same as $n$-vector, relative to $R$. Thus, $R$ is not a resolving set of $\Lambda$.

Case 2: if $\left|R_{1}\right|=\left|R_{2}\right|$ and there are vertices $x, y \in R_{1}$, such that $x$ is adjacent to $y$ in $\Lambda$, then there are vertices $u, v \in V_{1}-R_{1}$ such that $u$ is adjacent to $v$ in $\Lambda$. Therefore, the metric representation of the vertices $u, v \in V(\Lambda)-R$ is the same as $n$-vector, relative to $R$. Thus, $R$ is not a resolving set of $\Lambda$.

Case 3: now, let $\left|R_{1}\right|=\left|R_{2}\right|$, and suppose that, for all the vertices $x, y$ in $R_{1}$, we have $x$ is not adjacent to $y$ in $\Lambda$, that is, $d(x, y)=2$. Also, for all the vertices $u, v$ in $R_{2}$, we have $u$ is not adjacent to $v$ in $\Lambda$, that is, $d(u, v)=2$. We may assume that $R_{1}=\left\{a, a^{2}, \ldots, a^{n / 2}\right\}$ and $R_{2}=\left\{a b, a^{2} b\right.$, $\left.\ldots, a^{n / 2} b\right\}$. So, we can assume that an arranged subset $R$ of vertices in graph $\Lambda$ is $R=\left\{a, a^{2}, \ldots, a^{n / 2} ; a b\right.$, $\left.a^{2} b, \ldots, a^{n / 2} b\right\}$. Hence, $V(\Lambda)-R=\left\{a^{n+2 i / 2}, \ldots, a^{n} ; a^{n+2 i / 2}\right.$ $\left.b, \ldots, a^{n} b\right\}$, for $1 \leq i \leq n / 2$.

Therefore, the metric representations of the vertices $a^{(n+2) / 2}, a^{(n+4) / 2}, \ldots, a^{n} ; a^{(n+2) / 2} b, a^{(n+4) / 2} b, \ldots, a^{n} b \in V(\Lambda)-R$ relative to $R$ are the $n$-vectors:

$$
\begin{aligned}
r\left(a^{(n+2) / 2} \mid R\right) & =(1,2,2, \ldots, 2 ; 1, \ldots, 1), r\left(a^{(n+4) / 2} \mid R\right) \\
& =(2,1,2, \ldots, 2 ; 1, \ldots, 1), \ldots, r\left(a^{n} \mid R\right) \\
& =(2,2, \ldots, 1 ; 1,1, \ldots 1)
\end{aligned}
$$

and $\quad r\left(a^{(n+2) / 2} b \mid R\right)=(1, \ldots, 1 ; 1,2,2, \ldots, 2), \quad r\left(a^{(n+4) / 2} b \mid R\right)$ $=(1, \ldots, 1 ; 2,1,2, \ldots, 2), \ldots, r\left(a^{n} b \mid R\right)=(1,1, \ldots 1 ; 2,2, \ldots, 1)$.

Thus, all the vertices in $V(\Lambda)-R$ have different representations relative to $R$. This implies that $R$ is a resolving set of $\Lambda$. 
Theorem 2. If $n$ is an even integer greater than or equal 4 and $\Lambda=\operatorname{Cay}\left(\mathbb{D}_{2 n}, \Psi\right)$ is a Cayley graph on the dihedral group $\mathbb{D}_{2 n}$, where $\Psi$ is defined already, then the cardinality of minimum doubly resolving set of $\Lambda$ is $n$.

Proof. By the previous theorem, we know that the arranged subset $R=\left\{a, a^{2}, \ldots, ; a b, a^{2} b, \ldots, a^{n / 2} b\right\}$ of vertices in the graph $\Lambda$ is a resolving set for $\Lambda$. We show that the subset $R$ is a doubly resolving set of $\Lambda$. It is sufficient to show that, for two vertices $u$ and $v$ in graph $\Lambda$, there are vertices $x, y \in R$ such that $d(u, x)-d(u, y) \neq d(v, x)-d(v, y)$. Consider two vertices $u$ and $v$ of $\Lambda$. By the following cases, we can be concluded that the minimum cardinality of a doubly resolving set of $\Lambda$ is $n$.

Case 1: consider a pair of distinct vertices $u, v \in \Lambda$ such that $u, v \in R$. Then, the length of a shortest path from $u$ to $v$ is $d(u, v)=1$ or 2 . Let $u$ and $v$ be two vertices in $R$ such that a shortest path from $u$ to $v$ in graph $\Lambda$ is $d(u, v)=1$. We may assume that $u=a$ and $v=a b$. Hence, by taking $x=a \in R$ and $y=a^{n / 2} \in R$, we have $-2=0-2=d(u, x)-d(u, y) \neq d \quad(v, x)-d(v, y)=$ $1-1=0$. Therefore, the vertices $x$ and $y$ of $R$ doubly resolve $u, v$. Now, let $u, v$ be two vertices in $R$ such that a shortest path from $u$ to $v$ in graph $\Lambda$ is $d(u, v)=2$. We may assume that $u=a$ and $v=a^{n / 2}$. Hence, by taking $x=a \in R \quad$ and $y=a b \in R$, we have $-1=0-1=d(u, x)-d(u, y) \neq d(v, x)-d(v, y)=2-$ $1=1$. Therefore, the vertices $x$ and $y$ of $R$ doubly resolve $u, v$.

Case 2: consider a pair of distinct vertices $u, v \in \Lambda$ such that $u \in R$ and $v \notin R$. Then, the length of a shortest path from $u$ to $v$ is $d(u, v)=1$ or 2. Suppose a pair of distinct vertices $u \in R$ and $v \notin R$ are adjacent in graph $\Lambda$, that is, $d(u, v)=1$. We may assume that $u=a$ and $v=a^{n+2 / 2}$. Hence, by taking $x=a \in R$ and $y=a b \in R$, we have $-1=0-1=d(u, x)-d(u, y) \neq d \quad(v, x)-d(v, y)=$ $1-1=0$. Therefore, the vertices $x$ and $y$ of $R$ doubly resolve $u, v$. Now, suppose a pair of distinct vertices $u \in R$ and $v \notin R$ are not adjacent in graph $\Lambda$, that is, $d(u, v)=2$. We may assume that $u=a$ and $v=a^{n}$. Hence, by taking $x=a \in R$ and $y=a b \in R$, we have $-1=0-1=d(u, x)-d(u, y) \neq d(v, x)-d$

$(v, y)=2-1=1$. Therefore, the vertices $x$ and $y$ of $R$ doubly resolve $u, v$.

Case 3: consider a pair of distinct vertices $u, v \in \Lambda$ such that $u \notin R$ and $v \notin R$. Then, the length of a shortest path from $u$ to $v$ is $d(u, v)=1$ or 2 . We can show that the subset $R$ of vertices in graph $\Lambda$ is a doubly resolving set of $\Lambda$. Because by Theorem 1 , we can be concluded that $V(\Lambda)-R$ is also a resolving set of $\Lambda$.

Lemma 1. If $n$ is an even integer greater than or equal 4 and $\Lambda=\operatorname{Cay}\left(\mathbb{D}_{2 n}, \Psi\right)$ is a Cayley graph on the dihedral group $\mathbb{D}_{2 n}$, where $\Psi$ is defined already, then the subset $R=\left\{a, a^{2}, \ldots, a^{n / 2} ; a b, a^{2} b, \ldots, a^{n / 2} b\right\}$ of vertices in graph $\Lambda$ is not a strong resolving set of $\Lambda$.
Proof. We know that the arranged subset $R=\left\{a, a^{2}, \ldots, a^{n / 2} ; a b, a^{2} b, \ldots, a^{n / 2} b\right\}$ of vertices in graph $\Lambda$ is a resolving set for $\Lambda$ of size $n$. Now, let $V(\Lambda)=V_{1} \cup V_{2}$, where $V_{1}=\left\{a, a^{2}, \ldots, a^{n}\right\}, V_{2}=\left\{a b, a^{2} b, \ldots, a^{n} b\right\}$, and $R=R_{1} \cup R_{2}$, where $R_{1}=\left\{a, a^{2}, \ldots, a^{n / 2}\right\} \quad$ is a subset of $V_{1}$ and $R_{2}=\left\{a b, a^{2} b, \ldots, a^{n / 2} b\right\}$ is a subset of $V_{2}$. Consider two vertices $u, v$ in $\Lambda$ such that $u, v \in V_{1}-R_{1}$ and $u$ is not adjacent to $v$ in $\Lambda$, that is, $d(u, v)=2$. In the following cases, we show that there is not $w \in R$ such that $w$ is strongly resolve vertices $u$ and $v$. For every vertex $w \in R$, we have $w \in R_{1}$ or $w \in R_{2}$.

Case 1: if $w \in R_{1}$, then the length of a shortest path from $u$ to $w$ is $d(u, w)=1$ or 2 and length of a shortest path from $v$ to $w$ is $d(v, w)=1$ or 2 . Note that, if $d(u, w)=1$, then $d(v, w)=2$. Therefore, $w$ is not strongly resolve vertices $u$ and $v$. In particular, if $d(u, w)=2$, then $d(v, w)=1$ or 2 , and hence, $w$ is not strongly resolve vertices $u$ and $v$ because $d(u, v)=2$.

Case 2: if $w \in R_{2}$, then the length of a shortest path from $u$ to $w$ is $d(u, w)=1$ and length of a shortest path from $v$ to $w$ is $d(v, w)=1$. Therefore, $w$ is not strongly resolve vertices $u$ and $v$.

Theorem 3. If $n$ is an even integer greater than or equal 4 and $\Lambda=\operatorname{Cay}\left(\mathbb{D}_{2 n}, \Psi\right)$ is a Cayley graph on the dihedral group $\mathbb{D}_{2 n}$, where $\Psi$ is defined already, then the strong metric dimension of $\Lambda$ is $2 n-2$.

Proof. Let $V(\Lambda)=V_{1} \cup V_{2}$, where $V_{1}=\left\{a, a^{2}, \ldots, a^{n}\right\}$ and $V_{2}=\left\{a b, a^{2} b, \ldots, a^{n} b\right\}$. It is not hard to see that if $n \geq 4$, then the size of largest clique in the graph $\Lambda$ is 4 . Moreover, we know that the subset $N=\left\{a^{n}, a^{n / 2} ; a^{n} b, a^{n / 2} b\right\}$ of vertices in $\Lambda$ is a clique in the graph $\Lambda$. Now, let the subset $S$ of vertices in $\Lambda$ be $S=V(\Lambda)-N$. In the following cases, we show that the subset $S$ of vertices in $\Lambda$ is not a strong resolving set of $\Lambda$.

Case 1: let $u=a^{n}$ and $v=a^{n / 2}$. We know that $d(u, v)=$ 1 and $u, v \in V_{1}$, and hence, for every $w \in S$ such that $w \in V_{2}$, we have $d(u, w)=1$ and $d(v, w)=1$. Thus, $w$ is not strongly resolve vertices $u$ and $v$.

Case 2: now, let $u=a^{n}$ and $v=a^{n / 2}$. We know that $d(u, v)=1$ and $u, v \in V_{1}$, and hence, for every $w \in S$ such that $w \in V_{1}$, we have $d(u, w)=2$ and $d(v, w)=2$. Thus, $w$ is not strongly resolve vertices $u$ and $v$.

Therefore, the subset $S$ of the vertices in graph $\Lambda$ is not a strong resolving set of $\Lambda$. From the above cases, we can be concluded that the minimum cardinality of a strong resolving set for $\Lambda$ must be $2 n-2$.

\section{Conclusion}

Computing resolving parameters of a graph is an NP-hard problem. In this study, we considered a class of Toeplitz graphs and we denoted by $T_{2 n}(W)$ so that they are isomorphic to the Cayley graph $\Lambda=\operatorname{Cay}\left(\mathbb{D}_{2 n}, \Psi\right)$, which is defined already. In fact, this class of graphs is vertex transitive, and by calculating the spectrum of the adjacency 
matrix related with them, we showed that this class of graphs cannot be edge transitive. Also, we proved that this class of graphs cannot be distance regular, and because of the difficulty of the computing resolving parameters of a class of graphs which are not distance regular, we regarded this as justification for our focus on some resolving parameters. In particular, we determined the minimal resolving set, doubly resolving set, and strong metric dimension for this class of graphs.

\section{Data Availability}

No data were used to support this study.

\section{Conflicts of Interest}

The authors declare that there are no conflicts of interest regarding the publication of this paper.

\section{Acknowledgments}

This work was supported in part by Natural Science Fund of Education Department of Anhui Province, under Grant KJ2020A0478.

\section{References}

[1] C. Godsil and G. Royle, Algebraic Graph Theory, Springer, New York, NY, USA, 2001

[2] F. Harary and R. A. Melter, "On the metric dimension of a graph,” Combinatoria, vol. 2, pp. 191-195, 1976.

[3] P. J. Slater, "Leaves of trees," in Proceedings of the 6th Southeastern Conference on Combinatorics, Graph Theory and Computing, Boca Raton, FL, USA, 1975.

[4] R. F. Bailey and P. J. Cameron, "Base size, metric dimension and other invariants of groups and graphs," Bulletin of the London Mathematical Society, vol. 43, no. 2, pp. 209-242, 2011.

[5] Z. Beerliova, F. Eberhard, T. Erlebach et al., "Network discovery and verification," IEEE Journal on Selected Areas in Communications, vol. 24, no. 12, pp. 2168-2181, 2006.

[6] M. Imran, M. K. Siddiqui, and R. Naeem, "On the metric dimension of generalized petersen multigraphs," IEEE Access, vol. 6, pp. 74328-74338, 2018.

[7] S. Khuller, B. Raghavachari, and A. Rosenfeld, Localization in Graphs, University of Maryland, College Park, MD, USA, 1994.

[8] J.-B. Liu and A. Zafari, "Computing minimal doubly resolving sets and the strong metric dimension of the layer Sun graph and the Line Graph of the Layer Sun Graph," Complexity, vol. 2020, Article ID 6267072, 8 pages, 2020.

[9] T. Vetrík and A. Ahmad, "Computing the metric dimension of the categorial product of some graphs," International Journal of Computer Mathematics, vol. 94, no. 2, pp. 363-371, 2015.

[10] P. Halmos, A Hilbert Space Problem Book, American Book Company, Knoxville, TN, USA, 1967.

[11] J.-B. Liu, M. F. Nadeem, H. M. A. Siddiqui, and W. Nazir, "Computing metric dimension of certain families of Toeplitz graphs," IEEE Access, vol. 7, pp. 126734-126741, 2019.

[12] R. van Dal, G. Tijssen, Z. Tuza, J. A. A. van der Veen, C. Zamfirescu, and T. Zamfirescu, "Hamiltonian properties of
Toeplitz graphs," Discrete Mathematics, vol. 159, no. 1-3, pp. 69-81, 1996.

[13] R. F. Bailey, "The metric dimension of small distance-regular and strongly regular graphs," Australas. J. Combin, vol. 62, no. 1, pp. 18-34, 2015.

[14] J.-B. Liu, A. Zafari, and H. Zarei, "Metric dimension, minimal doubly resolving sets, and the strong metric dimension for jellyfish graph and cocktail party graph," Complexity, vol. 2020, pp. 1-7, Article ID 9407456, 2020.

[15] N. L. Biggs, Algebraic Graph Theory, Cambridge University Press, New York, NY, USA, 1993.

[16] A. E. Brower, A. M. Cohen, and A. Neumaier, Distance Regular Graphs, Springer, Berlin, Germany, 1989.

[17] J. Cáceres, C. Hernando, M. Mora et al., "On the metric dimension of Cartesian products of graphs," SIAM Journal on Discrete Mathematics, vol. 21, no. 2, pp. 423-441, 2007.

[18] A. Sebö and E. Tannier, "On metric generators of graphs," Mathematics of Operations Research, vol. 29, no. 2, pp. 383393, 2004.

[19] F. Harary and A. J. Schwenk, "Which graphs have integral spectra?" Lecture Notes in Mathematics, vol. 406, pp. 45-51, 1974.

[20] L. W. Beineke and R. J. Wilson, Topics in Algebraic Graph Theory, Cambridge University Press, Cambridge, UK, 2004.

[21] S. M. Mirafzal and A. Zafari, "On the spectrum of a class of distance-transitive graphs," Electronic Journal of Graph Theory and Applications, vol. 5, no. 1, pp. 63-69, 2017.

[22] R. Frucht, "On the groups of repeated graphs," Bulletin of the American Mathematical Society, vol. 55, pp. 418-420, 1949.

[23] S. M. Mirafzal and A. Zafari, "An interesting property of a class of circulant graphs," Journal of Mathematics, vol. 2017, 4 pages, 2017.

[24] W. T. Tutte, Connectivity in Graphs, University of Toronto Press, Toronto, Canada, 1966. 